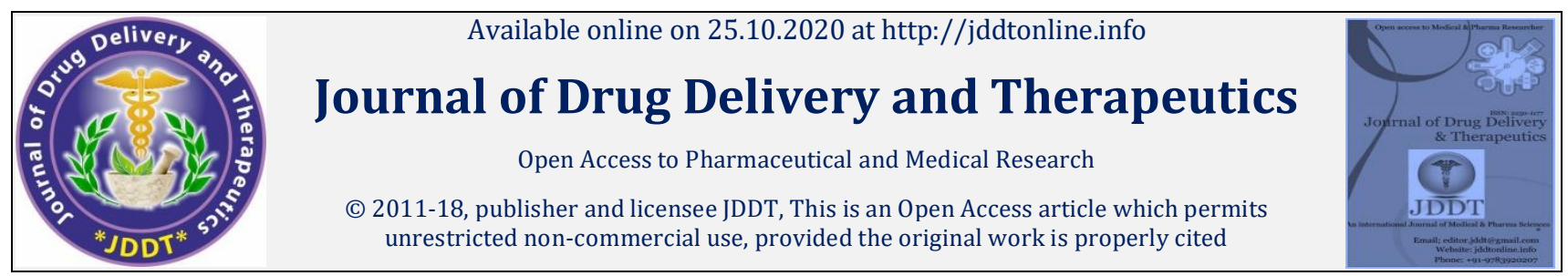

Open $\odot$ Access

Research Article

\title{
Therapeutic Efficacy of Moxifloxacin Mucoadhesive Hydrogel for Bacterial Keratitis
}

\author{
Nipuna Kumar Bhoi ${ }^{*}$, A. K. Singhai ${ }^{1}$, Gaurav Jain'1, Subhendu S. Mishra ${ }^{2}$ \\ ${ }^{1}$ Lakshmi Narain College of Pharmacy, Bhopal, M.P, India \\ ${ }^{2}$ Kalinga Biomedical Research Institute \& Training Establishment, Sambalpur, Odisha, India
}

\begin{abstract}
Bacterial keratitis is a hypothetically devastating corneal infection due to the opportunity of fast development; corneal devastation either to be completed in 24-48 hours with even more contagious bacterial aetiological agents. Moxifloxacin mucoadhesive Hydrogel was prepared by using polymer Hydroxy Propyl Methyl Cellulose E50 LV by hydration method. Moxifloxacin was dissolved in small quantity of water and Benzalkonium Chloride was added to the Polymer solution. The formulations were evaluated for clarity, pH measurement, spread-ability test, drug content estimation, viscosity study, in vitro diffusion study and antibacterial activity. The developed formulation exhibits the sustained release over a period of 10 hour. The optimized formulation was further evaluated with antimicrobial activity. The results of the in-vitro antimicrobial activity of hydrogel were satisfactory.
\end{abstract}

Keywords: Corneal Infection, Hydrogel, Moxifloxacin, invitro release.

Article Info: Received 17 Aug 2020; Review Completed 29 Sep 2020; $\quad$ Accepted 12 Oct 2020; Available online 25 Oct 2020

Cite this article as:

Bhoi NK, Singhai AK, Jain G, Mishra SS, Therapeutic Efficacy of Moxifloxacin Mucoadhesive Hydrogel for Bacterial Keratitis, Journal of Drug Delivery and Therapeutics. 2020;10(5-s):177-185 http://dx.doi.org/10.22270/jddt.v10i5s.4369

*Address for Correspondence:

Mr. Nipuna Bhoi, M. Pharm Scholar, Lakshmi Narain College of Pharmacy, Bhopal, M.P, India

\section{INTRODUCTION:}

Keratitis, a spectrum of ocular diseases, affecting cornea and pathogenically resulting from bacterial, fungal, and protozoal etiologic organisms can potentially cause ocular morbidity and disability ${ }^{1}$. Mostly corneal infection is reliant on three factors: the degree of pathogenicity of the etiologic organism, the underlying condition of the cornea, and the immunological state of the individual ${ }^{2}$.

It is a condition where the cornea the clear, round dome layer. The eye's iris and the pupil become swollen or reddened and painful. It is also defined as a corneal ulcer. Contact lens wearers essential to evoke that infectious keratitis ${ }^{3-6}$. The numeral of multi-resistant CoNS (Coagulase-negative staphylococci) recognized in patients with lively ocular blemish has also sustained to upsurge in topical years ${ }^{7}$. The clear diagnosis after bacterial keratitis rest on the size, locality, and depth of the ulcer, as well as on the prospects and the bacteria remoted8.Bacterial keratitis, due to its high rate and hypothetical hitches, is one of the extreme visual aggressive ocular infective pathologies. The avascular corneal stroma is especially vulnerable to bacterial infection. Corneal damages, which have been șated 250-1177 particularly, invasive pathogens such as Pseudomonas aeruginosa and Staphylococcus aureus ${ }^{9}$.

Mucoadhesion is thought to be caused by two mechanisms: the contact stage and the consolidation stage. Mucoadhesive that attaches to a mucus membrane may result in swelling of the formation. This condition leads to the spread of the formation along the surface of the mucosal layer. This may facilitate penetration of hydrogel inside mucosal layers ${ }^{10}$.

Moxifloxacin Mucoadhesive hydrogel drug delivery system can enhance the bioavailability of therapeutic agents by avoiding drug degradation. Hydrophobic and hydrophilic molecules can be integrated inside a core-shell assembly while the surface can be further functionalized by ligands and other molecules to increase its cellular attachment. This system is suitable for multidrug encapsulation. The physicochemical properties of mucoadhesive systems (e.g., swelling, expanding, and shrinking), are advantageous to reaction with mucosal layers, allowing hydrogel to be internalized in tissues ${ }^{11-13}$.

The standard initial treatment consists of frequent instillation of eye drops containing a broad-spectrum antibiotic. However, poor retention of a drug (tear-out from 
the ocular cavity) and poor ocular bioavailability of drugs in form of a drop has remained one of the greatest challenges to treating these clinical conditions. It has the greatest importance in providing sustained ocular drug delivery. By exhibiting elastic properties, they resist ocular drainage of the drug leading to longer contact times.

The objective of the present investigation to formulate insitu gelling thermosensitive mucoadhesive hydrogel by an antibacterial agent, Moxifloxacin using a combination of Carbopol-934 with different mucoadhesive polymers such as HPMC and Sodium alginate to increase gel strength and adhesion force and thereby increased precorneal contact time and bioavailability of the drug.

\section{MATERIALS AND METHODS:}

\section{Materials:}

Moxifloxacin is a gift sample from Alkem pharmaceutical company, Sikkim (India). Carbopol - 934was purchased fromAlpha chemika Pvt. Ltd, Mumbai. HPMC E50LV was purchased from Avra Synthesis Pvt. Ltd. Hyderabad. Sodium Alginate, Benzalkonium Chloride, Buffer capsule were procured from Research-lab fine chem industries Mumbai. Sodium hydroxide was purchased from HiMedia Laboratories Pvt. Ltd. of Mumbai.

\section{Methods:}

The formulation procedure for Moxifloxacin mucoadhesive Hydrogel drug delivery system was charted below. Formulation ingredients with their extents were as specified in table-1. The buffer salts were dissolved in $75 \mathrm{~mL}$ of purified water, Hydroxy Propyl Methyl Cellulose E50 LV was added \& allowed for hydration, Carbopol - 934 were sprinkled over the solution and allowed for hydration overnight. The solution was stirred with an overhead stirrer with $1000 \mathrm{rpm}$. Moxifloxacin was dissolved in a small quantity of water and Benzalkonium Chloride was added to the Polymer solution. Then make up the volume up to 100 $\mathrm{mL}$, then the solution was filtered through Whatman filter paper 14

Table:1-Composition of Mucoadhesive Hydrogel

\begin{tabular}{|c|c|c|c|c|c|c|c|}
\hline Formulations & $\begin{array}{c}\text { Moxifloxacin } \\
\text { (g) }\end{array}$ & $\begin{array}{c}\text { Carbopol- } \\
\mathbf{9 3 4} \mathbf{( g )}\end{array}$ & $\begin{array}{c}\text { HPMC } \\
\text { E50LV }\end{array}$ & $\begin{array}{c}\text { Sodium } \\
\text { Alginate }\end{array}$ & $\begin{array}{c}\text { Benzalkonium } \\
\text { Chloride }\end{array}$ & $\begin{array}{c}\text { Sodium } \\
\text { Hydroxide }\end{array}$ & $\begin{array}{c}\text { Distilled } \\
\text { water } \\
\text { (ml) }\end{array}$ \\
\hline F1 & 0.4 & 0.2 & 0.1 & - & 1 & 0.01 & 100 \\
\hline F2 & 0.4 & 0.3 & 0.2 & - & 1 & 0.01 & 100 \\
\hline F3 & 0.4 & 0.4 & 0.3 & - & 1 & 0.01 & 100 \\
\hline F4 & 0.4 & 0.2 & 0.4 & - & 1 & 0.01 & 100 \\
\hline F5 & 0.4 & 0.3 & - & 0.1 & 1 & 0.01 & 100 \\
\hline F6 & 0.4 & 0.4 & - & 0.2 & 1 & 0.01 & 100 \\
\hline F7 & 0.4 & 0.2 & - & 0.3 & 1 & 0.01 & 100 \\
\hline F8 & 0.4 & 0.3 & - & 0.4 & 1 & 0.01 & 100 \\
\hline
\end{tabular}

\section{Evaluation of Hydrogel}

\section{a) Clarity and Visual appearances:}

Clarity and visual appearance can be determined by observing transparent or white particles against the black background \& dark or black particles against the white background.

\section{b) pH determination:}

The $\mathrm{pH}$ of the gel formulations was determined by using a $\mathrm{pH}$ meter. For $\mathrm{pH}$ determination, $1 \%$ of hydrogel formulation in deionized water was prepared and $\mathrm{pH}$ was determined 15 .

\section{c) Spread-ability Test:}

Spreadability can be determined by applying the gel over an even surface and observed for the gritty nature of the hydrogel if present 16 .

\section{d) Viscosity Study:}

The viscosity of gels was determined by using a Brook Field viscometer DV-II model. T-Bar spindles in combination with a Heli path stand were used to measure the viscosity.

\section{e) Drug content:}

From the developed moxifloxacin hydrogel formulation, $1 \mathrm{ml}$ of suspension is a mixture in the $10 \mathrm{ml}$ of $1.2 \mathrm{pH}$ buffer. The amount of insulin was determined using a UV spectrophotometer at $272 \mathrm{~nm}^{17}$.

\section{f) Measurement of the gel strength:}

The gel sample of $25 \mathrm{~mL}$ was put in a $50 \mathrm{~mL}$ graduated cylinder. A weight of $14.33 \mathrm{~g}$ was placed on the gel surface.
The gel strength, which is an expression for the ophthalmic gel at physiological temperature, was determined by the time in seconds required by the weight to penetrate $5 \mathrm{~cm}$ into the gel. All measurements were performed in triplicate $(n=3)$.

\section{g) In-vitro diffusion study:}

An in-vitro drug release study was performed utilizing altered Franz diffusion cells. The dialysis layer (Hi-Media, Molecular weight 5000 Daltons) was put among receptor and donor compartments. In-situ gel proportional to $100 \mathrm{mg}$ of moxifloxacin hydrogel was set in the donor compartment and the receptor compartment was loaded up with phosphate buffer, $\mathrm{pH}$ 7.4. The dispersion cells were kept up at $37 \pm 0.5^{\circ} \mathrm{C}$ with blending at $50 \mathrm{rpm}$ all through the investigation. At various time interim, $5 \mathrm{ml}$ of aliquots were pulled back from the recipient compartment through the side cylinder and examined for medication content by UV Visible spectrophotometer ${ }^{18}$.

\section{Sterility study:}

The well diffusion method was used to determine the antibacterial activity of the mucoadhesive hydrogel using the standard procedure. The drug used in standard preparation was Moxifloxacin of IP grade. The antibacterial activity was performed by using $24 \mathrm{hr}$ culture of Staphylococcus aureus. There was 3 concentration used which are $1 \%, 2 \%$ and $3 \%$ for antibiogram studies. The plates were incubated at $37^{\circ} \mathrm{C}$ for 24 hours and then examined for clear zones of inhibition around the wells impregnated with a particular concentration of the drug ${ }^{19}$. 


\section{RESULTS AND DISCUSSION:}

The characterizations of the hydrogel, Physical appearance of the formulations were light white and clear. Table-2 shows the gelling capacity of all formulations, which is depicted as + (gel forms in 40 to 50 seconds and dissolves rapidly) and ++ (gel forms within 60 seconds and remains stable for 3 hours. The gelling capacity increases with increasing concentration of gelling agents both at a higher and lower concentration. The $\mathrm{pH}$ value of all the prepared formulations ranged from $6.9 \pm 0.03$ to $7.3 \pm 0.06$, which is considered acceptable to avoid the risk of irritation upon application to the eye. The viscosity of the different formulations was compared as shown in Table-2. It was shown that the viscosity was an increase when the concentration of Carbopol increased. Eight formulations were evaluated for drug content and in vitro dissolution study. The drug content was in between $95.42 \pm 0.04$ to $98.46 \pm 0.04$ in different formulation. All the data was reported in table- 2 .
The invitro cumulative \% drug release of the mucoadhesive hydrogel of moxifloxacin was $82.89 \pm 0.049 \%$ to $91.89 \pm 0.021$ $\%$ at the end of $10 \mathrm{~h}$, respectively as shown in Table- 3 and Fig-4. The kinetic release profile of hydrogel shows that formulation MHG6 was the best formulation and it follows the Higuchi release profile. In vitro release pattern was fitted to the Higuchi model Which recommends that Ocular in situ hydrogel released drug in a controlled release manner in 10 hours.

The antibacterial activity of the mucoadhesive hydrogel was assayed to determine the zone of inhibition against different bacteria. that antimicrobial activity of at three different concentrations $1 \%, 2 \%$, and $3 \%$ by disc diffusion method. It indicated that the formulation displayed a variable degree of antimicrobial activity. The inhibitory effect increases when the increase of the formulation concentration.[Table - 5]

Table 2: Evaluation of Moxifloxacin in situ hydrogel Gel Formulations

\begin{tabular}{|c|c|c|c|c|c|c|}
\hline Code & Clarity & pH & $\begin{array}{c}\text { Spreadability } \\
\text { (gm.cm/sec) }\end{array}$ & $\begin{array}{c}\text { Viscosity } \\
\text { (Cps) }\end{array}$ & $\begin{array}{c}\text { Drug Content } \\
\text { (\%) }\end{array}$ & Gel strength \\
\hline MHG1 & Clear & $7.3 \pm 0.06$ & $10.83 \pm 0.75$ & $6514 \pm 1.25$ & $96.61 \pm 0.02$ & + \\
\hline MHG2 & Clear & $6.9 \pm 0.06$ & $11.53 \pm 0.42$ & $5428 \pm 0.83$ & $95.42 \pm 0.04$ & + \\
\hline MHG3 & Clear & $7.1 \pm 0.06$ & $11.25 \pm 0.56$ & $5175 \pm 1.82$ & $98.15 \pm 0.01$ & ++ \\
\hline MHG4 & Clear & $7.2 \pm 0.03$ & $11.29 \pm 0.81$ & $7245 \pm 2.34$ & $96.51 \pm 0.08$ & + \\
\hline MHG5 & Clear & $7.1 \pm 0.05$ & $10.91 \pm 0.36$ & $5487 \pm 0.71$ & $98.14 \pm 0.04$ & + \\
\hline MHG6 & Clear & $6.9 \pm 0.03$ & $11.38 \pm 0.62$ & $6547 \pm 1.15$ & $97.45 \pm 0.07$ & ++ \\
\hline MHG7 & Clear & $7.3 \pm 0.05$ & $11.73 \pm 0.56$ & $5048 \pm 2.48$ & $98.46 \pm 0.04$ & ++ \\
\hline MHG8 & Clear & $7.3 \pm 0.04$ & $11.23 \pm 0.82$ & $7542 \pm 1.15$ & $96.76 \pm 0.04$ & + \\
\hline
\end{tabular}

Table 3: In-vitro drug release study of moxifloxacin

\begin{tabular}{|c|c|c|c|c|c|c|c|c|}
\hline \multicolumn{9}{|c|}{ Cumulative \% of drug release } \\
\hline $\begin{array}{c}\text { Time } \\
\text { (hour) }\end{array}$ & MHG1 & MHG2 & MHG3 & MHG4 & MHG5 & MHG6 & MHG7 & MHG8 \\
\hline $\mathbf{0}$ & 0 & 0 & 0 & 0 & 0 & 0 & 0 & 0 \\
\hline $\mathbf{1}$ & $8.27 \pm 0.028$ & $7.46 \pm 2.25$ & $10.24 \pm 0.037$ & $9.82 \pm 0.037$ & $12.21 \pm 0.077$ & $10.05 \pm 0.049$ & $9.53 \pm 0.045$ & $13.54 \pm 0.054$ \\
\hline $\mathbf{2}$ & $18.56 \pm 0.015$ & $20.56 \pm 1.13$ & $19.87 \pm 0.046$ & $18.56 \pm 0.083$ & $26.58 \pm 0.014$ & $25.45 \pm 0.061$ & $24.58 \pm 0.052$ & $23.56 \pm 0.046$ \\
\hline $\mathbf{3}$ & $23.58 \pm 0.011$ & $32.25 \pm 1.75$ & $30.25 \pm 0.075$ & $28.87 \pm 0.075$ & $35.45 \pm 0.073$ & $34.47 \pm 0.035$ & $33.65 \pm 0.068$ & $32.45 \pm 0.091$ \\
\hline $\mathbf{4}$ & $38.58 \pm 0.015$ & $42.56 \pm 1.08$ & $40.78 \pm 0.019$ & $38.78 \pm 0.093$ & $48.78 \pm 0.093$ & $47.98 \pm 0.044$ & $46.58 \pm 0.042$ & $45.58 \pm 0.083$ \\
\hline $\mathbf{6}$ & $56.89 \pm 0.018$ & $55.48 \pm 1.59$ & $53.47 \pm 0.078$ & $51.15 \pm 0.057$ & $63.45 \pm 0.051$ & $62.14 \pm 0.062$ & $60.14 \pm 0.073$ & $60.01 \pm 0.093$ \\
\hline $\mathbf{8}$ & $78.45 \pm 0.022$ & $72.45 \pm 2.57$ & $68.78 \pm 0.045$ & $65.56 \pm 0.077$ & $75.74 \pm 0.046$ & $74.56 \pm 0.071$ & $73.47 \pm 0.072$ & $72.56 \pm 0.069$ \\
\hline $\mathbf{1 0}$ & $91.89 \pm 0.021$ & $89.45 \pm 1.58$ & $85.45 \pm 0.067$ & $83.98 \pm 0.037$ & $87.56 \pm 0.043$ & $85.85 \pm 0.049$ & $82.89 \pm 0.049$ & $83.89 \pm 0.046$ \\
\hline
\end{tabular}

Table 4: Regression analysis data

\begin{tabular}{|c|c|c|c|c|}
\hline \multirow{2}{*}{ Code } & Zero Order & First Order & Higuchi's Model & Korsmeyer Peppas Equation \\
\cline { 2 - 5 } & $\mathbf{R}^{\mathbf{2}}$ & $\mathbf{R}^{\mathbf{2}}$ & $\mathbf{R}^{\mathbf{2}}$ & 0.699 \\
\hline MHG1 & 0.959 & 0.993 & 0.953 & 0.706 \\
\hline MHG2 & 0.968 & 0.983 & 0.968 & 0.681 \\
\hline MHG3 & 0.978 & 0.994 & 0.965 & 0.653 \\
\hline MHG4 & 0.968 & 0.981 & 0.951 & 0.693 \\
\hline MHG5 & 0.969 & 0.965 & 0.963 & $\mathbf{0 . 7 0 5}$ \\
\hline MHG6 & $\mathbf{0 . 9 7 3}$ & $\mathbf{0 . 9 7 9}$ & $\mathbf{0 . 9 7 8}$ & 0.680 \\
\hline MHG7 & 0.970 & 0.986 & 0.953 & 0.702 \\
\hline MHG8 & 0.977 & 0.991 & 0.962 & \\
\hline
\end{tabular}


Table 5: Zone of Inhibition of Formulated Hydrogel \& Marketed in situ Hydrogel

\begin{tabular}{|c|c|c|c|c|c|}
\hline \multirow{2}{*}{ S. No. } & \multirow{2}{*}{ Product Details } & \multicolumn{4}{|c|}{ Zone of inhibition by using S.aureus } \\
\cline { 3 - 6 } & & $\mathbf{0}$ & $\mathbf{1 \%}$ & $\mathbf{2 \%}$ & $\mathbf{3 \%}$ \\
\hline 1. & Moxifloxacin in situ Hydrogel & 0 & $9 \pm 0.57$ & $10 \pm 0.28$ & $12 \pm 0.47$ \\
\hline 2. & Marketed in situ Hydrogel & 0 & $8 \pm 0.76$ & $9 \pm 0.17$ & $9 \pm 0.87$ \\
\hline
\end{tabular}

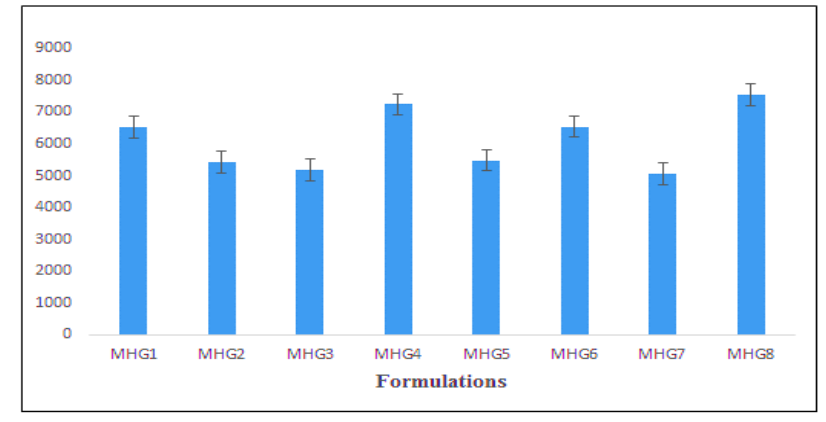

Fig-1 Viscosity of all formulation

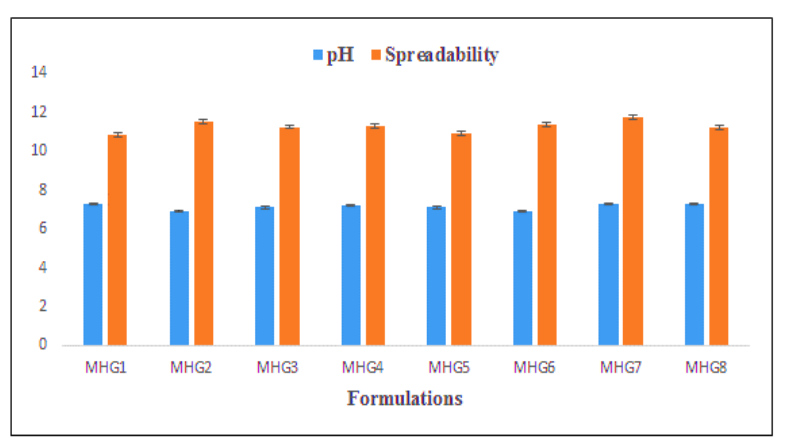

Fig-2 Spreadability and pH

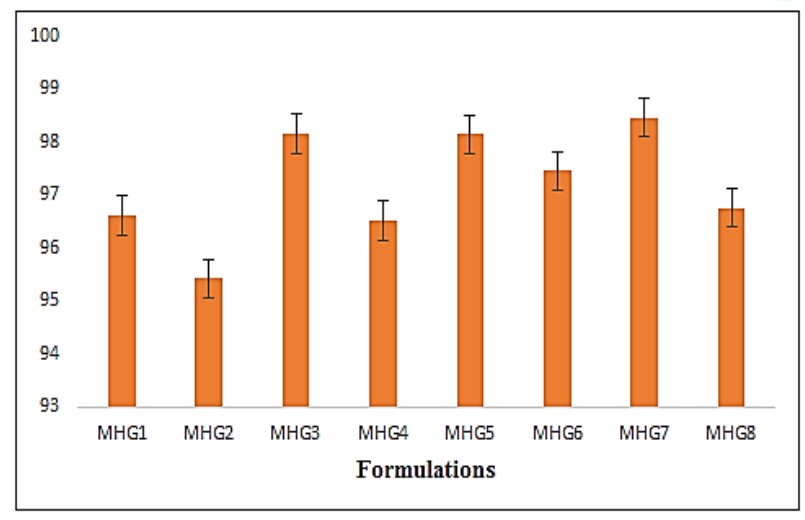

Fig-3 drug content of all formulation

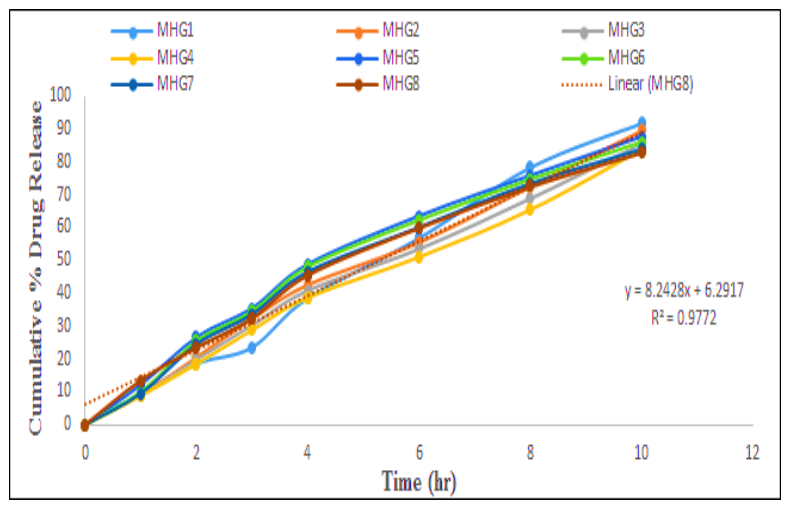

Fig-4 In-vitro release study

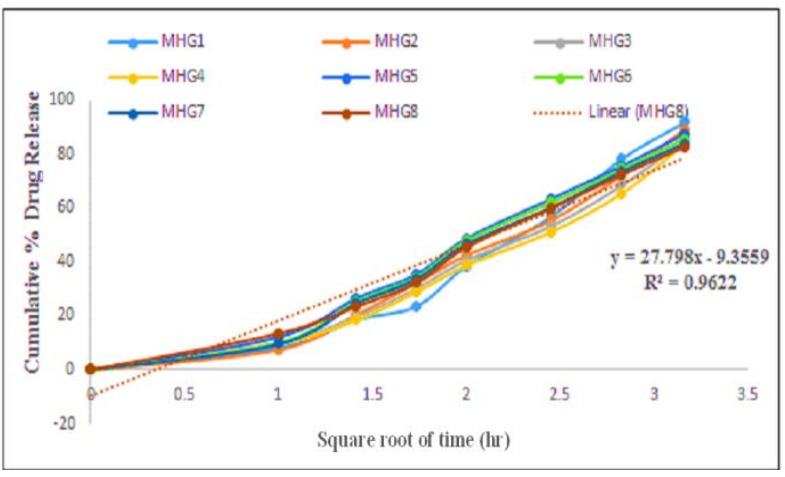

Fig-5 Zero-order release kinetic study

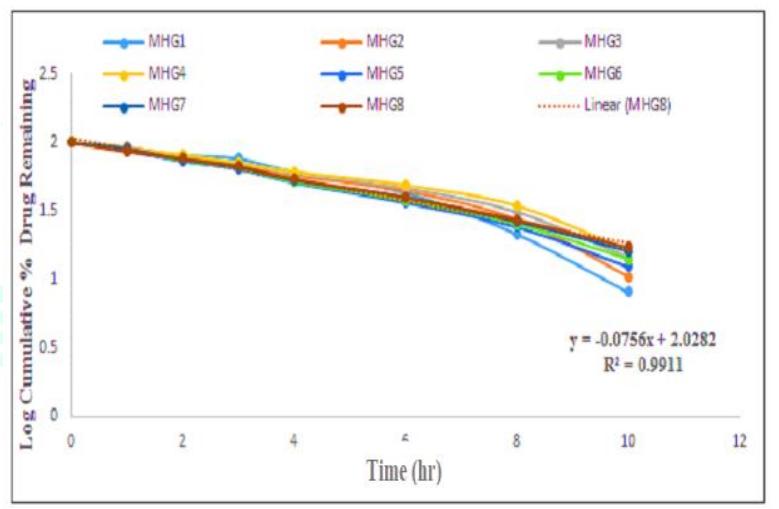

Fig-6 First order Kinetic Study

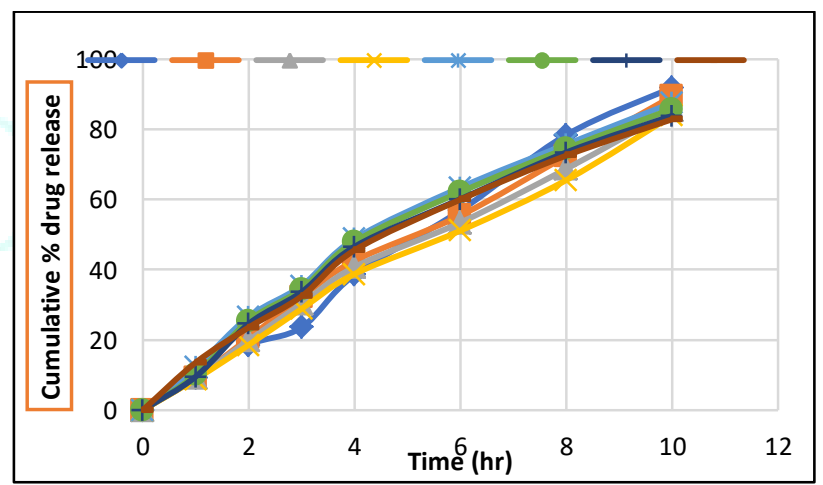

Fig-7 Higuchi Equation

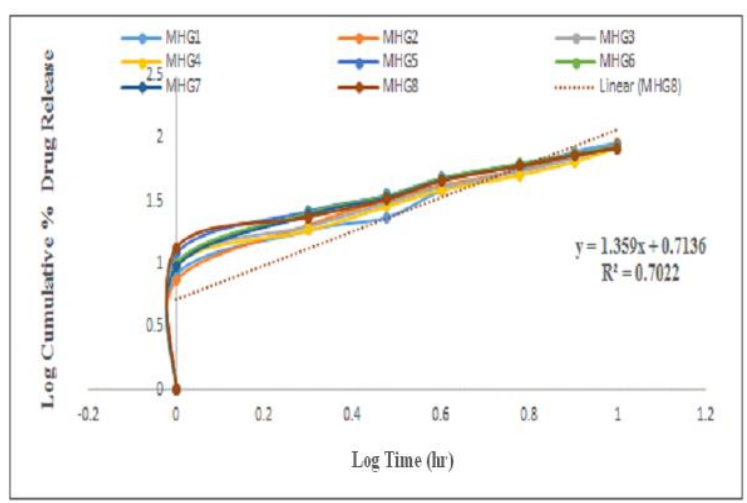

Fig-8 Korsmeyer-Peppas Equation

CODEN (USA): JDDTAO 


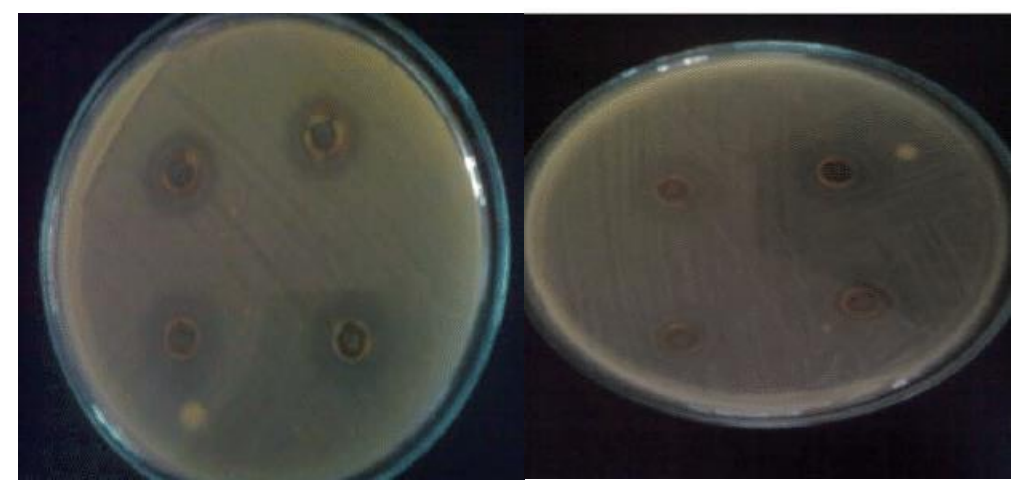

[Moxifloxacin in situ Hydrogel] [Marketed in situ Hydrogel]

Fig-9 Zone of Inhibition of Plate

\section{CONCLUSION:}

Moxifloxacin Hydrochloride is a broadspectrum fluoroquinolone antibiotic. Moxifloxacin binds besides inhibiting the bacterial enzymes' DNA gyrase (topoisomerase II) and topoisomerase IV resulting in inhibition of DNA replication, repair, and cell death in sensitive bacterial species. Hydrogel produced by mucoadhesive polymers has the advantage of being immobilized at the mucosal surface by an adhesion mechanism. The mucoadhesive action depends on the physical reaction of hydrogel moieties with the structure of mucosal content and results in the swelling and expansion of the hydrogel. The ophthalmic in situ hydrogel was formulated using Carbopol-934 (g), HPMC E50LV, and Sodium Alginate. The clarity of the prepared formulation was satisfactory. The $\mathrm{pH}$ was ranging between 6.9 \pm 0.03 to $7.3 \pm 0.06$. the drug content of the prepared formulation was satisfactory. The formulations of MHG1 to MHG8 showed sustained drug release for 10 hours. Formulation MHG6 showed the maximum sustained drug release. Based on drug content, $\mathrm{pH}$, Spreadability, and Invitro release data were found to be satisfactory. The results of the in-vitro antimicrobial activity of hydrogel were also satisfactory.

\section{Acknowledgments:}

Authors acknowledge to Alkem pharmaceutical company, Sikkim for Gift sample Moxifloxacin. The author is grateful to the Principal Dr. A.K. Singhai of Lakshmi Narain College of Pharmacy, Bhopal, and Professor Dr. Gaurav jain for providing necessary facilities and information.

\section{Conflicts of Interest:}

The authors declare that there is no conflict of interest regarding the publication of this paper.

\section{REFERENCES:}

1. Schaftenaar E, Peters RPH, Baarsma GS, Meenken C, Khosa NS and Getu S. () Clinical and corneal microbial profile of infectious keratitis in a high HIV prevalence setting in rural South Africa. Eur J Clin Microbiol Infect Dis.2016; 35:1403-9.

2. Robles-Contreras A, Perez-Cano HJ, Babayan-Sosa A, BacaLozada 0. Bacterial keratitis infection: A battle between virulence factors and the immune response, Common Eye Infections. Imtiaz Chaudhry, IntechOpen, DOI: 10.5772/52264.

3. Leck AK, ThomasPA, Hagan M.et al., Aetiology of suppurative corneal ulcers in Ghana and South India, and epidemiology of fungal keratitis. The British Journal of Ophthalmology.2002; 86 (11):1211-1215.
4. Pinna A, Zanetti S, Sotgiu M, Sechi LA, FaddaG, Carta F. Identification and antibiotic susceptibility of coagulase-negative staphylococci isolated in corneal/external infections. The British Journal of Ophthalmology.1999; 83(7):771-773.

5. ManikandanP, Bhaskar M, Revathy R, JohnRK, Narendran K, NarendranV. Speciation of coagulase-negative Staphylococcus causing bacterial keratitis.Indian Journal of Ophthalmology.2005; 53(1):59-60.

6. Ly AN, PhamJN, BadenochPR, et al. Bacteria commonly isolated from keratitis specimens retain antibiotic susceptibility to fluoroquinolones and gentamicin plus cephalothin," Clinical and Experimental Ophthalmology.2006; 34 (1):44-50.

7. JohnJF, HarvinAM. History and evolution of antibiotic resistance in coagulase-negative staphylococci: susceptibility profiles of new anti-staphylococcal agents. Therapeutics and Clinical Risk Management.2007; 3(6):1143-1152.

8. Schein OD, BuehlerPO, StamlerJF, VerdierDD, KatzJ. The impact of overnight wear on the risk of contact lens-associated ulcerative keratitis. Archives of Ophthalmology. 1994; 112 (2):186-190,.

9. Limberg MB. A review of bacterial keratitis and bacterial conjunctivitis. Am J Ophthalmol. 1991; 112:2S-9S.

10. Hägerström H.Edsman K. Strømme M. Low-frequency dielectric spectroscopy as a tool for studying the compatibility between pharmaceutical gels and mucus tissue. J. Pharm. Sci. 2003, 92:1869-1881.

11. Hanafy NAN, Quarta A, Ferraro MM, Dini L, Nobile C, De Giorgi ML. Carallo S, Citti C, Gaballo A, Cannazza G,et al. Polymeric Nano-MicellesasNovel Cargo-Carriers for LY2157299 LiverCancerCells Delivery. Int. J. Mol. Sci. 2018; 6:19.

12. Hanafy NAN, El-Kemary M, Leporatti S. Reduction diameter of CaCO3 crystals by using polyacrylic acid might improve cellular uptake of encapsulated curcumin in breast cancer. J. Nanomed. Res. 2018, 7:235-239.

13. Hanafy NA, DeGiorgi MLD, Nobile C, Rinaldi R, Leporatti S. Control of Colloidal CaCO3 suspension by using biodegradable polymers during fabrication. Beni-Suef Univ. J. Basic Appl. Sci. 2015; 4:60-70.

14. Preethi GB, Narendra E. Formulation and Evaluation of In Situ Mucoadhesive Ophthalmic Hydrogel for Sustained Delivery of Pefloxacin Mesylate. International Journal of Pharmacy and Pharmaceutical Sciences. 2015; 7(8):345-350.

15. Gokulgandhi MR, Parikh JR, Megha Barot M, Modi DM. A pH triggered in situ gel-forming ophthalmic drug delivery system for tropicamide. Drug Delivery Technology. 2007; 5:44-9.

16. Zhidong L, Jiawei L, Shufang N, Hui L, Pingtian D, Weisan P. Study of an alginate/HPMC based in situ gelling ophthalmic delivery system for gatifloxacin. Int J Pharm. 2006; 315:12-7.

17. Khanna R, Agarwal S P, Ahuja A. Preparation and evaluation of mucoadhesive buccal films of clotrimazole for oral Candida infections. Indian J Pharm Sci. 1997; 59:299-305.

18. Sultana Y, Aqil M, Ali A, Zafar S. Evaluation of carbopolmethylcellulose based sustained-release ocular delivery system for pefloxacin mesylate using rabbit eye model. Pharm Dev Technol. 2006; 11:313-9.

19. Desi HA, Bhalla HL. Preparation and evaluation of a new eye drop containing a combination of ciprofloxacin and dexamethasone. Indian Drugs. 2000; 37:112. 